Editorial

\title{
Acknowledgement to Reviewers of Languages in 2018
}

\section{Languages Editorial Office}

MDPI, St. Alban-Anlage 66, 4052 Basel, Switzerland; languages@mdpi.com

Published: 10 January 2019

Rigorous peer-review is the corner-stone of high-quality academic publishing. The editorial team greatly appreciates the reviewers who contributed their knowledge and expertise to the journal's editorial process over the past 12 months. In 2018, a total of 42 papers were published in the journal, with a median time to first decision of 37.5 days and a median time to publication of 133.5 days. The editors would like to express their sincere gratitude to the following reviewers for their cooperation and dedication in 2018:

$\begin{array}{ll}\text { Alba De La Fuente, Anahi } & \text { Chatterjee, Tridha } \\ \text { Alexiadou, Artemis } & \text { Chengappa, Shyamala. K. } \\ \text { Almenara, Erika } & \text { Chiari, Isabella } \\ \text { Andrews, Edna } & \text { Childers, Jane } \\ \text { Anton, Eneko } & \text { Chu, Mingyuan } \\ \text { Antoniou, Mark } & \text { Clark, Diane } \\ \text { Antonucci, Sharon } & \text { Clark, Eve V. } \\ \text { Ardila, Alfredo } & \text { Colantoni, Laura } \\ \text { Argyri, Froso } & \text { Couto, M Carmen Parafita } \\ \text { Arosio, Fabrizio } & \text { Cunliffe, Daniel } \\ \text { Backus, A. M. } & \text { Cuza, Alejandro } \\ \text { Balieiro Lodi, Ana Claudia } & \text { De Benito, Carlota } \\ \text { Belletti, Adriana } & \text { De La Fuente, Israel } \\ \text { Beltrán-Palanques, Vicent } & \text { den Dikken, Marcel } \\ \text { Benkato, Adam } & \text { Domínguez, Laura } \\ \text { Berry, Grant } & \text { Dubiel, Bozena } \\ \text { Bigelow, Ann E. } & \text { Dubinina, Irina } \\ \text { Borgonovo, Claudia } & \text { Dussias, Paola (Giuli) } \\ \text { Bouzouita, Miriam } & \text { Dyson, Bronwen } \\ \text { Bowles, Melissa } & \text { E. Requena, Pablo } \\ \text { Brandl, Anel } & \text { Elvira González, Javier } \\ \text { Brown, Esther } & \text { Escutia, Marciano } \\ \text { Brown, Joshua } & \text { Español-Echevarría, Manuel } \\ \text { Bultena, Sybrine } & \text { Faiq, Said } \\ \text { Canseco-González, Enriqueta } & \text { Fernández-Berkes, Eva } \\ \text { Carrol, Gareth } & \text { François, Thomas } \\ \text { Casasanto, Daniel } & \text { Fuchs, Robert } \\ \text { Casillas, Joseph } & \text { Gajek, Elżbieta } \\ \text { Castillo Lluch, Monica } & \text { Garayzábal-Heinze, Elena } \\ \text { Cazzoli, Marcela } & \text { Garnham, Alan } \\ \text { Celaya, M. Luz } & \text { Gerholm, Tove } \\ & \end{array}$


Gess, Randall

Giancaspro, David

Goldrick, Matt

Green, David

Hemforth, Barbara

Heredia, Roberto

Herschensohn, Julia

Hilpert, Martin

Hintz, Florian

Hirakawa, Makiko

Hiramoto, Mie

Hogue, Lawrence

Homan, Christopher

Hostetter, Autumn

Izwaini, Sattar

Jegerski, Jill

Jerger, Susan

Kaushanskaya, Margarita

Kazanas, Stephanie

Klassen, Rachel

Klee, Carol A

Koops, Christian

Koro, Ruth

Koronkiewicz, Bryan

Kouega, Jean-Paul

Krausneker, Verena

Han, Min Kyung

Labelle, Marie

Larkosh, Christopher

Leonetti, Manuel

Lepic, Ryan

Linck, Jared A.

Lipski, John

Llama, Raquel (Sweden)

Llama, Raquel (Canada)

Lleó, Conxita

Luo, Xuanmin

Maher, Kate

Mathy, Fabien

Matsuda, Kenjiro

Mattock, Karen

McClure, William

Meakins, Felicity

Medvedeva, Olga

Miyagawa, Shigeru

Müller, Natascha

Munarriz, Amaia

Muñoz Pérez, Carlos

Muntendam, Antje
Muysken, Pieter

Napoli, Donna

Occhino, Corrine

O'Neill, Paul

Orozco, Rafael

Ortega, Gerardo

Ortiz Ciscomani, Rosa María

Ortiz-Preuss, Elena

O'Shannessy, Carmel

Oudgenoeg-Paz, Ora

Ozturk, Ozge

Palacios, Azucena

Papadima-Sophocleous, Salomi

Peluso Crespi, Leonardo

Pérez-Leroux, Ana T.

Peristeri, Eleni

Perpinan, Silvia

Pescarini, Diego

Piantadosi, Steven T.

Piasecki, Anna

Pirvulescu, Mihaela

Pladevall, Elisabet

Pons Rodríguez, Lola

Prevost, Philippe

Rafat, Yasaman

Reed, Lisa

Reiners, Torsten

Repetto, Claudia

Requena, Pablo E.

Roberge, Yves

Roca Urgell, Francesc

Rodríguez Arancón, Pilar

Rodríguez Molina, Javier

Roeper, Thomas

Rossi, Eleonora

Rubio, Fernando

Samburskiy, Denis

Sanfelici, Emanuela

Sato, Takeshi

Schneider, Jason

Sewell, Andrew

Sleeman, Petra

Snape, Neal

Stadthagen-González, Hans

Steele, Jeffrey

Stewart, Jesse

Struiksma, Marijn

Thomas, Michael 
Tokowicz, Natasha

Torgersen, Eivind Nessa

Torrego, Esther

Torrens, Vincent

Trentman, Emma

Tschichold, Cornelia

Tsimpli, Ianthi Maria

Valdés Kroff, Jorge

Vales, Catarina
Van Gelderen, Elly

Vender, Maria

Venturi, Giulia

Wang, Yan

White, Lydia

Wrembel, Magdalena

Zamuner, Tania

Zhao, Cecilia

(C) 2019 by the author. Licensee MDPI, Basel, Switzerland. This article is an open access article distributed under the terms and conditions of the Creative Commons Attribution (CC BY) license (http://creativecommons.org/licenses/by/4.0/). 